\title{
Stroop and picture-word interference are two sides of the same coin
}

\author{
Leendert van MaAnen, Hedderik van RiJn, ANd Jelmer P. Borst \\ University of Groningen, Groningen, The Netherlands
}

\begin{abstract}
This article presents a cognitive model that reconciles a surprising observation in the picture-word interference (PWI) paradigm with the general notion that PWI is a form of Stroop interference. Dell'Acqua, Job, Peressotti, and Pascali (2007) assessed PWI using a psychological refractory period (PRP) paradigm, and concluded that the locus of interference in PWI is during the perceptual encoding stage. Stroop interference, on the other hand, is generally attributed to response selection. Based on these findings it was argued that PWI is not a Stroop effect. The present article discusses an alternative interpretation of these results. We assume that both effects are caused by the same interference mechanism, but that the processing speed associated with the different stimuli (colors vs. words) accounts for the previously reported differences. We support this argument by presenting a single computational model that accounts for both PWI and Stroop phenomena in single task and PRP settings.
\end{abstract}

Over the years, the idea that the picture-word interference (PWI) effect and the Stroop effect are two manifestations of the same process has gained wide support. For instance, MacLeod's influential review on the Stroop effect (MacLeod, 1991) also discusses PWI, and even lists the PWI task in his list of "eighteen major empirical results that must be explained by any successful account of the Stroop effect" (MacLeod, 1991, Appendix B, p. 203). Recently, Dell'Acqua, Job, Peressotti, and Pascali (2007) have argued that the underlying processes of the PWI and Stroop effects are different, challenging the assumption of a single underlying process. In this article, we will present a computational model that, on the basis of a single process, can explain both the traditional phenomena discussed by MacLeod (1991) and the data of Dell'Acqua et al. (2007).

In the Stroop paradigm, participants are presented with a word written in a specific color and are instructed to either read the word or name the color the word is printed in (MacLeod, 1991; Stroop, 1935). A typical Stroop experiment consists of three conditions: a congruent condition, in which the word and the color refer to the same color concept (e.g., the word "red" written in red ink); an incongruent condition, in which the word and the color refer to different color concepts (e.g., the word "red" written in green ink); and a neutral condition, in which only the text or the color is displayed. This is usually operationalized by presenting a set of Xs in red ink (for color naming) or the word red printed in black ink (for word reading).
Three experimental findings in a Stroop study are extremely robust. First, color naming in the incongruent condition is slower than color naming in the neutral condition. That is, participants required to name the color of the ink are slower if the word describes a different color than if they have to name the color of a row of Xs. This is often referred to as semantic interference, since many accounts of this effect assume that the relation in meaning between the color of the ink and the word itself causes the interference (e.g., Cohen, Dunbar, \& McClelland, 1990; W. R. Glaser \& Glaser, 1989; Klein, 1964). A second finding in the Stroop literature is that naming the color of the ink of a word that describes the same color (congruent condition) is faster than in the neutral condition. This will be referred to as semantic facilitation (but see MacLeod \& MacDonald, 2000 , for an alternative explanation of semantic facilitation based on accidental reading of the word). A third important observation is that both semantic interference and semantic facilitation disappear when participants are not instructed to name the color of the stimulus but instead are asked to pronounce the word, regardless of the color the word is printed in. This so-called Stroop asynchrony is often explained by the difference in processing speed between colors and words (e.g., Cohen et al., 1990; Roelofs, 1992), or the difference in automaticity between color naming and word reading (e.g., Lovett, 2005).

The PWI task typically has a setup similar to that of the Stroop task. Participants are presented with a picture on 
which a word is superimposed and are instructed to either name the picture or read the word. As in the Stroop task, semantic interference, semantic facilitation, and Stroop asynchrony are usually observed in the PWI task (W. R. Glaser \& Düngelhoff, 1984). Semantic interference is observed if participants are required to name a picture on which a category-member word of that picture is superimposed (e.g., a picture of a dog with the word cat in it), as their responses are slower compared to the condition in which a picture without a superimposed word has to be named, or to the condition in which the superimposed word is completely unrelated (e.g., a picture of a dog with the word desk superimposed). Semantic facilitation is observed if, in contrast, the picture and the word refer to the same concept (e.g., a picture of a cat with the word cat superimposed); that is, the participants are faster. The Stroop asynchrony can be observed in the disappearance of these effects if the task is to read the word instead of naming the picture.

At a more general level, these effects are often aggregated in terms of a single Stroop or PWI effect. This more general effect is the difference in performance between trials in which an interfering stimulus is present and trials in which one is not. For the Stroop task, this entails the difference between trials in which a color word is written in ink of a different color and trials in which a color word is written in similar-colored ink. For the PWI task, the effect is the difference between trials in which the picture is presented with a semantically related but different word superimposed and trials in which the picture and word are completely unrelated.

Given the similarity of the tasks, it is not surprising that both tasks have often been explained in similar terms. In fact, in our previous work, in which we presented a computational model of PWI, we took for granted that PWI is an instance of the Stroop effect (van Maanen \& van Rijn, 2007b). Other computational models also propose a single mechanism that underlies both PWI and Stroop performance (e.g., Cohen et al., 1990; Lovett, 2005; Roelofs, 1992, 2003).

Most theoretical accounts of Stroop-like effects have focused on finding the locus of the interference effect in the mental processing stream. For instance, many theorists assume that interference is caused by a competition between different response options (e.g., Cohen et al., 1990; Dyer, 1973; Roelofs, 2003, for the Stroop effect, and W. R. Glaser \& Glaser, 1989; Kuipers, La Heij, \& Costa, 2006; Lovett, 2005; Roelofs, 1992, for PWI). In this view, interference is caused by an increased difficulty of selecting the appropriate response in the incongruent condition. Often, this has been attributed to either a difference in the speed of processing between the stimulus dimensions or to a difference in automaticity between the stimulus dimensions (MacLeod, 1991).

Some theoretical accounts assume an interference effect during an early stage. In particular, Dell'Acqua et al. (2007) argued that the PWI effect is caused by a competition that occurs before an appropriate response is selected. As for the Stroop effect, many studies now suggest that it may be manifested at multiple stages (e.g., De Houwer, 2003; Risko, Schmidt, \& Besner, 2006; Schmidt \& Cheesman, 2005; Van Veen \& Carter, 2005).

Recently it has been suggested that the Stroop effect and PWI are not caused by the same process (Dell'Acqua et al., 2007). In particular, it has been argued that the loci of the two interference effects in the mental processing stream differ. Many studies suggest that the locus of the Stroop effect is on the level of response selection (e.g., Fagot \& Pashler, 1992; Kuipers et al., 2006; MacLeod, 1991; Roelofs, 2003). That is, Stroop interference occurs because an incorrect response possibility triggered by the distracting feature of the stimulus (the word) interferes with the correct response triggered by the target feature (the color). On the basis of a psychological refractory period experiment, Dell'Acqua et al. argued that this is not the case for PWI. Their result suggests that PWI is on the level of the perceptual encoding. Based on these results, Dell'Acqua et al. concluded that, although the effects in both tasks seem similar, they are in fact caused by different mechanisms.

This article presents a single computational cognitive model of both the Stroop and the PWI effect, reconciling the recently observed differences between Stroop and PWI with the general view that PWI is an instance of the Stroop effect. In this model, both Stroop and PWI effects are accounted for by the same process. Whether the interference effects are manifested early in the mental processing stream during a perceptual encoding stage or late during response selection is determined by the stimulus type (i.e., previously unseen pictures vs. easily identifiable colors). In other words, the model provides additional evidence for the view that the underlying process that causes interference in both tasks is the same (cf. Cohen et al., 1990; Lovett, 2005; Roelofs, 1992, 2003), while still being able to explain the observation that participants respond differently to Stroop and PWI stimuli under certain circumstances (Dell' Acqua et al., 2007).

\section{Analyzing the Locus of Interference}

To analyze the time course of the Stroop effect, Fagot and Pashler (1992, Experiment 7) studied whether the Stroop effect would persist in a psychological refractory period (PRP) design. In a PRP design, participants are required to perform two tasks concurrently. The main manipulation in PRP designs is an asynchrony in onset between both tasks. Usually, the instruction is to first give the response associated with the stimulus that was displayed first. If the stimulus onset asynchrony (SOA) between tasks is relatively long, the processing of the first task is finished before the stimulus of the second task is presented. On the other hand, if the SOA is short, processing associated with the first stimulus might not be finished when the second stimulus appears. The typical observation with short SOAs is that the response to the second task is delayed (Telford, 1931). This delay is often interpreted as evidence for a bottleneck in processing. The central bottleneck theory assumes that there exists a processing stage during which only a single process can 
proceed concurrently (e.g., Pashler, 1994). Therefore, the task presented second will be delayed by the processing of the first task.

Fagot and Pashler (1992) used the PRP paradigm in the context of the Stroop task. They presented participants with a simple tone classification task as primary task, and a Stroop task as secondary task. Fagot and Pashler hypothesized that if the Stroop effect-operationalized as the latency difference between an incongruent Stroop stimulus and a congruent Stroop stimulus - was caused by the perceptual encoding of the stimulus, the Stroop effect would disappear at short SOAs. This would be the result of a response selection delay in the second task. This delay creates a gap in processing of the second task in which the interference could be resolved. Fagot and Pashler found no effect of SOA on the magnitude of the Stroop effect, which they interpreted as evidence that the locus of the Stroop effect is located relatively late in the stream of mental processing. This finding, they argued, is in line with a response selection account for the locus of the Stroop effect, because response selection is late in the mental processing stream.

In a similar experiment, Dell'Acqua et al. (2007) answered the same question for the PWI effect. Dell'Acqua et al. conducted an experiment very similar to the experiment run by Fagot and Pashler (1992), but instead of a Stroop task, participants were presented with a PWI task. In this experiment, the interference was operationalized as the latency difference between a semantically related word-picture pair and an unrelated word-picture pair. Interestingly (and surprisingly), SOA mediated the PWI effect: Shorter SOAs were associated with smaller PWI effects. This indicates that the inference originates from the initial stages of processing, since, with short SOAs, most interference is captured in the PRP-induced delay; with long SOAs, however, the delay does not account for all interference. This finding is in line with the view that the locus of PWI is early, possibly during perceptual encoding of the stimulus.

Based on this result, Dell'Acqua et al. (2007) argued that the difference between their findings and Fagot and Pashler's (1992) is "incompatible with the often reiterated principle that the PWI effect comes about for limitations of the cognitive system that are analogous to those causing the Stroop effect" (Dell'Acqua et al., 2007, p. 720). They concluded that their analysis "favor[s] an interpretation of the present findings that points to the functional dissociation of the sources of Stroop and PWI effects" (Dell'Acqua et al., 2007, p. 722).

The PRP studies by Dell'Acqua et al. (2007) and Fagot and Pashler (1992) may be accounted for by two explanations. The first possibility is the one advocated by Dell'Acqua et al.: Stroop and PWI are two different effects. The other possibility is that the interference in Stroop and PWI is distributed over multiple stages. However, the amount of interference per stage may differ between Stroop and PWI. This explanation is in line with findings in the Stroop literature that Stroop interference may be caused by stimulus-related competition as well as by response competition (e.g., De Houwer, 2003; Risko et al., 2006; Schmidt \& Cheesman, 2005).

We will support this second explanation by presenting a cognitive model that can account for reaction time (RT) data of both PWI and Stroop experiments. The sole difference between the models is in the speed of processing for the perceptual input (i.e., previously unseen pictures vs. easily identifiable colors). Next, we will demonstrate that the model accounts for the response time patterns of Stroop and PWI tasks under PRP conditions. Since these fits are obtained with one model, in which only one parameter is manipulated, this argues against the claim that the differential findings for Stroop and PWI favor a dissociation of the sources of Stroop and PWI effects.

\section{THE MODEL}

The cognitive model that we will describe is an integrated cognitive model (Gray, 2007b) of the task, implemented in a previously validated cognitive architecture (Taatgen \& Anderson, 2008). We will simulate the complete process involved in the task, from the presentation of the stimulus up to the participant's response, resulting in quantitative predictions of RT data.

The model is implemented in the cognitive architecture ACT-R (Anderson, 2007). ACT-R assumes that specialized modules process different kinds of information. For instance, a visual module handles visual perception, and a motor module executes motor commands. Other modules that will play a role in the model described below are the declarative module, used for storing and accessing information in declarative memory, the speech module for speech output, the aural module for auditory perception, and a goal module for keeping track of goals and intentions (see Anderson, 2007; Taatgen, van Rijn, \& Anderson, 2007, for extensive descriptions of the identified modules). A central production rule system integrates the information made available by the different modules and issues new instructions to those modules. The production rule system communicates with the different modules through a set of interfaces called buffers. Behavior in ACT-R emerges from the selection and subsequent execution of production rules that consist of simple condition-action pairs. If the information present in the buffers matches the conditions of a production rule, that rule may be selected to execute its actions. Production rule actions consist of operations on the buffer contents, such as a request for new information from declarative memory, or a request for pressing a button on a keyboard.

Declarative information in ACT-R is represented in symbolic entities called chunks. Chunks represent simple facts, such as The capital of The Netherlands is Amsterdam, or The object I am attending to is green. Note that where the first example is a typical semantic memory fact, the second example represents a visually observable feature of the world as might be present in the visual buffer. All chunks in an ACT-R model have an activation level that reflects the likelihood that they will be needed in the near future. The activation of a chunk depends on 
two components: the chunk's history of usage (Anderson \& Schooler, 1991) and the current context (Anderson \& Milson, 1989). The activation of a chunk is the main determiner of the time it takes to retrieve that chunk from memory. All other things being equal, the higher the activation of a chunk, the faster it will be retrieved.

Because Stroop tasks and PWI tasks usually involve well-known colors and words, and pictures of well-known objects, we will assume that the history component of the activation is approximately equal for all chunks as well as stable over the time span of a single experiment. For the context component of the activation, we will adopt a more fine-grained model that will be discussed later. This context component will be the main determiner for the RT differences that our model will display.

The model's behavior is determined by the interaction between specified production rules, chunk retrievals, and the task setup. In terms of response latency (the usual dependent measure in Stroop and PWI tasks), the model's behavior is the result of an aggregation of the timing of the subprocesses, such as the execution time of the production rules, and the time associated with module-specific operations, such as declarative retrievals and buttonpresses (cf. Donders, 1868/1969; Sternberg, 1969). However, although internally most module actions are executed sequentially, the modules themselves operate in parallel. For example, while the visual system is busy with the perceptual processes involved in perceiving a new stimulus, production rules might initiate a request for the retrieval of a fact from declarative memory without disturbing the perceptual process. Critically, however, the modules cannot execute multiple operations in parallel. Thus, if two tasks require retrieval from declarative memory, one of the tasks has to wait until the declarative module is finished with the request of the other task. This seriality will be critical in the explanation of PWI and Stroop performance. Although this seriality has not been specifically designed to account for performance in tasks in which scarce resources determine behavior, it has been successfully applied in many different experimental domains, such as attentional blink (Taatgen, Juvina, Schipper, Borst, \& Martens, 2009), language development (Hendriks, van Rijn, \& Valkenier, 2007; van Rij, van Rijn, \& Hendriks, 2009), temporal cognition (van Rijn \& Taatgen, 2008; for reviews, see Byrne \& Anderson, 2001; Salvucci \& Taatgen, 2008).

The model presented here, in line with the proposal of Dell'Acqua et al. (2007), assumes that both the Stroop task and PWI consist of three main stages: the perceptual encoding stage, the response selection stage, and the response execution stage. During the perceptual encoding stage, the stimulus features are transferred to the visual buffer. During the response selection stage, a chunk that reflects the syntactic properties of the response (i.e., a lemma; Levelt, Roelofs, \& Meyer, 1999) is retrieved from declarative memory. In the response execution stage, the model retrieves a motor program associated with the retrieved response, and this motor program is executed.
During each stage, one or more memory retrievals take place. The durations of these retrievals are the main determiners of the response latency. Given that we assumed that all chunks are equally active, all differences in activation are driven by the context activation. If the current state of the system is favorable for a chunk requested from memory, the chunk will be retrieved faster than in a less favorable context. This context phenomenon determines whether semantic interference or semantic facilitation is observed.

\section{A More Fine-Grained Account of Context Effects}

The current declarative retrieval module in ACT-R can account for many memory-related phenomena (e.g., Anderson, Bothell, Lebiere, \& Matessa, 1998; Taatgen \& Anderson, 2002; van Rijn \& Anderson, 2003). Although the module accurately predicts the duration of memory retrievals, ACT-R does not provide an account of what happens during memory retrievals. This is especially problematic in tasks with multiple stimuli presented at short SOAs, since the first, not-yet-completed retrieval is influenced by a second process. This dependency cannot be explained by default ACT-R (van Maanen, 2009; van Maanen \& van Rijn, 2007b).

To overcome this issue, we proposed an adaptation of the declarative retrieval mechanism in the cognitive architecture ACT-R to account for the time course of memory retrieval on short time scales (van Maanen \& van Rijn, 2007b). This retrieval account, or RACE/A (retrieval by accumulating evidence in an architecture), predicts what happens during the actual retrieval process. RACE/A is driven by two key assumptions: (1) The activation of one chunk is determined (in part) by the activation of other chunks; and (2) the activation of one chunk relative to the activation of other chunks determines the likelihood that it will be retrieved.

The first assumption represents the notion that the relevance of information is context-dependent, even when this context is not yet available at a symbolic level (i.e., accessible to the production system). This is reflected, for instance, in subliminal priming studies, in which a related prime decreases the response latency on a target stimulus, even when participants were not consciously aware of the prime (Marcel, 1983). We operationalized this by adopting a spreading activation strategy (Collins \& Loftus, 1975), in which increased activation of one chunk increases the activation of related chunks.

$$
C_{i}(t)=\alpha C_{i}(t-1)+\beta \sum_{j \in k} C_{j}(t-1) S_{j i}
$$

Equation 1 implements this assumption. The equation reflects how the activation of a chunk $\left[C_{i}(t)\right]$ accumulates during retrieval. The activation at time $t$ depends on the previous activation of that chunk $\left[C_{i}(t-1)\right]$, as well as on additional spreading activation $\left[C_{j}(t-1) S_{j i}\right]$ from other chunks $(k)$. This includes the chunks that are available through perceptual processing. The spreading activation is mediated by the associative strength between two chunks $\left(S_{j i}\right)$, such that strongly associated chunks exchange more activation than loosely associated chunks. $\alpha$ and $\beta$ are 
scaling parameters that determine the relative contributions of both components. Because $\alpha$ is set to a value in the range $(0,1)$, it can be interpreted as temporal decay of activation. The accumulated activation thus decays after a retrieval has been attempted.

The second assumption states that the relative activation of a chunk determines the likelihood that that chunk will be retrieved. This assumption reflects the insight that if multiple memory representations are relevant, responding becomes more difficult (Luce, 1986). Following ACT-R, the activation of a chunk determines the likelihood that it will be needed in the near future. However, RACE/A extends the default ACT-R equations to take the activation of competing chunks into account. The activation levels of competing chunks are accounted for by taking the ratio of activation of the to-be-retrieved chunk (chunk $i$ in Equation 2) to the sum of activations of other relevant chunks (chunks $j$ in Equation 2; cf. Luce, 1986; Roelofs, 1992). In the present model, the relevant chunks are all those chunks that match the criteria specified by a retrieval request.

If the ratio specified in Equation 2 crosses a threshold $(\theta$, the retrieval ratio), the relative activation of the chunk in the denominator (chunk $i$ ) warrants the retrieval of that chunk. As soon as a chunk passes this threshold, RACE/A returns that chunk as the result of the retrieval process.

$$
\frac{e^{A_{i}}}{\sum_{j} e^{A_{j}}} \geq \theta
$$

On the basis of Equations 1 and 2, we have provided quantitative predictions for variants of both PWI (van Maanen \& van Rijn, 2007b) and the Stroop task (van Maanen \& van Rijn, 2007a). The PWI model focused on the effect of SOA differences; the Stroop model fitted a data set in which the distractors were presented subliminally.

Besides a theory of memory retrieval (RACE/A), which is the core of our modeling efforts in this article, a theory of perceptual encoding is also needed to study the Stroop and PWI effects. Similar to RACE/A, we deviate here from the theory currently implemented in ACT-R because it is too high-level for our purposes.

\section{Perceptual Encoding}

Before the processing of task-relevant information can commence, visual or auditory information has to be made available to central cognition. In both the PWI and Stroop tasks, all information is presented visually. In ACT-R, the perceptual encoding process results in a chunk entering the visual buffer and thus becoming available for further processing. By default, it is assumed that this process takes a fixed $85 \mathrm{msec}$ (Anderson, 2007). Although this is a sensible number when the details of the perceptual processing are less relevant for the task under study, the emphasis on perceptual encoding in Stroop and PWI tasks requires a more detailed account. However, it should be noted that our implementation of a perceptual encoding process should not be considered a complete theoretical account of perceptual processes. Rather, our implementa- tion should be considered a functional description, aimed at differentiating between the effects on the encoding time caused by different stimulus features (cf. Gray, 2007a).

We assume that features of the stimulus become available during the first stages of perceptual encoding (cf. feature integration theory-Treisman \& Gelade, 1980). Based on these features, chunks in declarative memory compete for retrieval. This process is implemented using the RACE/A mechanism: The visual features spread activation to chunks that represent concepts likely to be needed in the context of those features. Thus, concepts that relate to the visual features of the present stimulus receive activation, whereas concepts that do not relate do not receive activation. When a concept's activation results in a retrieval ratio larger than the threshold (see Equation 2), that concept is made available for nonvisual cognition as the result of visual processing. So, instead of a fixed duration for perceptual encoding, we have implemented perceptual encoding as a combination of a feature integration and selection process with a variable duration that depends on the characteristics of the input.

The variation in encoding time between different stimuli originates from two sources. First, since the relative activation of a chunk determines whether it will be encoded, the activation of other chunks in declarative memory partly determines the encoding time. Second, the speed by which activation spreads from features to chunks will be different for different types of stimuli (e.g., a color patch is easier to recognize than a complex line drawing). Therefore, a parameter is introduced that reflects the speed and strength of spreading activation from features to chunks, to account for the different encoding times associated with different types of stimuli (Dell'Acqua, Lotto, \& Job, 2000; Rossion \& Pourtois, 2004). Although our encoding account is much simpler than existing models of perceptual encoding (e.g., Itti \& Koch, 2001; Treisman \& Gelade, 1980; Wolfe, 1994) it provides the necessary detail to account for the perceptual processing in the PWI and Stroop tasks.

In the Stroop task, the color feature of the stimulus spreads activation to a concept representing that color, and the text feature of the stimulus spreads activation to the lemma associated with that word. For the PWI task, the features representing the line drawing spread activation to the concept chunk representing the content of the picture, whereas the word spreads activation to a lemma. Since word reading is faster than color naming (M. O. Glaser \& Glaser, 1982), more activation spreads from the text feature to the lemma representing the associated word than from the color feature to the associated color lemma.

In addition, we assume for the present model that line drawings are encoded slower than color patches. This assumption is supported by studies that show faster naming for sequences of color patches than for sequences of images (e.g., Denckla \& Rudel, 1976; Vukovic, Wilson, \& Nash, 2004).

In the model, these differences are operationalized by different settings for the parameter that reflects the speed of perceptual processing. In the remainder of this article, 
we will demonstrate that the interference dynamics observed in Stroop and PWI PRP tasks might be caused by differences in this perceptual processing speed.

\section{Simulation 1: A Single Model for PWI and Stroop}

Before discussing our model of the Stroop and PWI effects under PRP conditions, we will first discuss the model's performance in non-PRP Stroop or PWI trials. Note that although this section focuses on a Stroop trial, exchanging references to color dimensions with references to pictures results in a description of how the model would perform a PWI trial.
As soon as visual features become available to the model, activation is spread to the chunks associated with that visual information. The color features spread activation to a conceptual chunk representing that color, whereas the textual features spread activation to a lemma.

When a chunk representing either the lemma or the concept crosses the retrieval ratio threshold, a second stage is initiated. Since the availability of a lemma is a prerequisite for starting the response execution stage, the model directly enters this stage when the lemma crosses the threshold. In this stage, the model retrieves a word form and the response is uttered. If the color has to be named, the model waits until a concept is selected on the basis of the visual input, after which a retrieval is initiated to retrieve
A

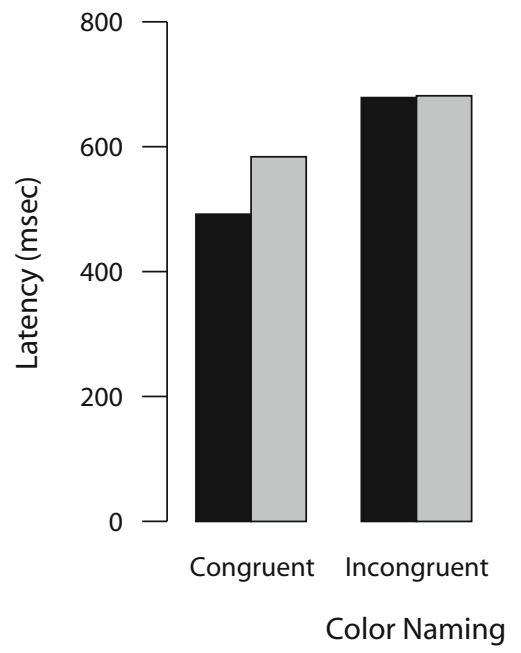

Stroop

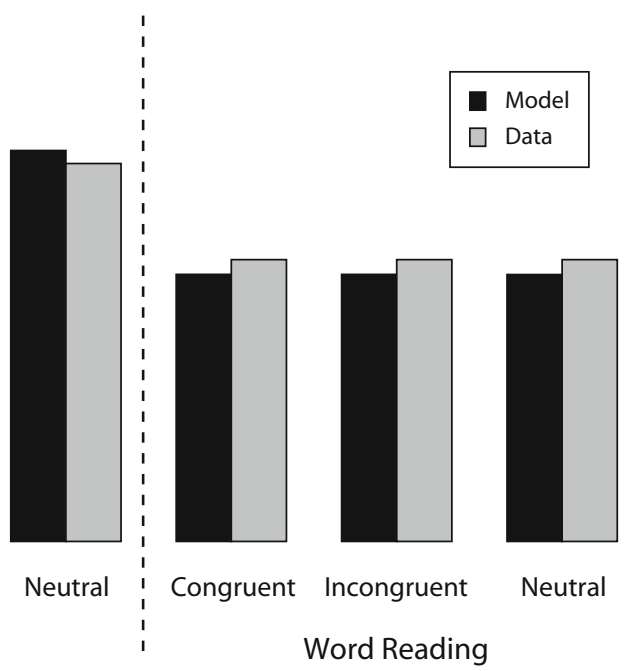

Picture-Word Interference

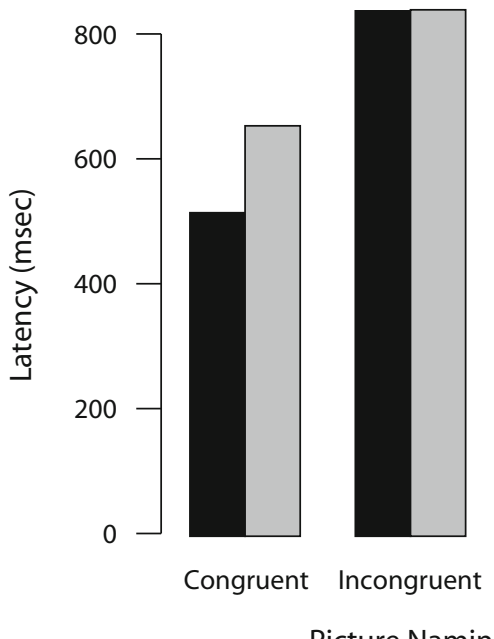

Picture Naming
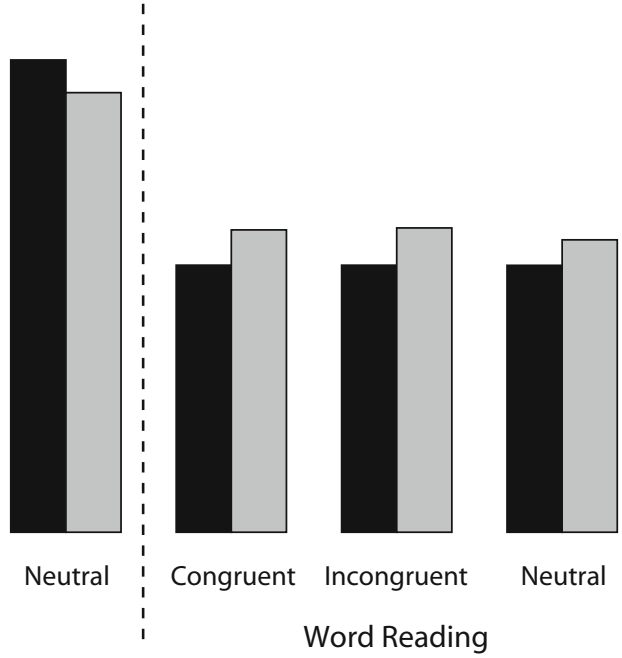

Congruent Incongruent

Neutral

Word Reading

Figure 1. Data and model for the Stroop task (A) and PWI (B). The Stroop data are from M. O. Glaser and Glaser, 1982 (Experiment 1, for SOA = 0); the PWI data are from W. R. Glaser and Düngelhoff, 1984 (Experiment 1, for SOA = 0). 
the lemma associated with the concept. However, because word reading is a highly trained and automated process, the word activates its associated lemma, even if the model expects a color concept. Because of this activation, and the earlier discussed retrieval ratio, color naming will show interference from the presented word, whereas word reading will show less interference. Note that the model described so far is very similar to models of the Stroop and PWI tasks presented by Roelofs $(1992,2003)$. In some cases, the visual word representation might have spread so much activation that the word-based lemma chunk exceeds the color-based lemma chunk in activation. In these situations, an incorrect lemma will be retrieved. To account for this, the model checks if the meaning of the lemma is consistent with the stimulus color (cf. van Rijn \& Anderson, 2004; other models of the Stroop task that incorporate a similar strategy include Altmann \& Davidson, 2001; Juvina \& Taatgen, 2009). If the correct lemma has been retrieved, the model continues with the response execution. If not, the model tries to retrieve another lemma. The response selection stage is the same for the word reading task: The model retrieves a word form and utters a response.

\section{Simulation Results and Discussion}

Figure 1A presents the fit of the Stroop model for both the color naming task and the word reading task (RMSE $\left.=33 \mathrm{msec}, R^{2}=.93\right)$ on the data from M. O. Glaser and Glaser (1982, Experiment 1, for SOA =0). The model captures both Stroop interference in the incongruent color naming condition and facilitation in the congruent color naming condition. In addition, the Stroop asynchrony between color naming and word reading can be observed. Given our hypothesis that the Stroop effect and PWI are manifestations of the same process, the challenge is to demonstrate that both effects can be fitted with the same model. Figure 1B presents the model fit on PWI RT data (W. R. Glaser \& Düngelhoff, 1984, Experiment 1, for SOA = 0). Similar to the fit of the Stroop task, all the behavioral patterns (interference, facilitation, and asynchrony) are captured by the model (RMSE $=60 \mathrm{msec}$, $R^{2}=.85$ ). The only difference between the two simulations underlying Figure 1 is a single parameter that controls the speed of processing of the stimulus adjusted to reflect the differences in stimuli between the two tasks: The processing speed was set lower for the picture-word stimulus than for the color-word stimulus, to reflect that visual processing of pictures in this particular task was slower than visual processing of colors.

Following our assumption that semantic interference is an effect of competition during the retrieval of declarative facts, we analyzed the amount of interference by comparing the duration of declarative memory retrievals between conditions. Thus, in our model, interference constitutes the difference in retrieval times. Figure 2 presents the difference in memory retrieval time between the model's incongruent and control conditions for both the Stroop task and the PWI task. Following others (e.g., Fagot \& Pashler, 1992; Ferreira \& Pashler, 2002; Jolicœur \& Dell'Acqua, 1998; McCann \& Johnston, 1992; Pashler, 1994; Van Selst \& Jolicœur, 1997), we assume that the bottleneck that Fagot and Pashler (1992) and Dell'Acqua et al. (2007) hypothesized is located after the perceptual encoding stage. Figure 2 shows the proportion of interference located before and after the hypothesized bottleneck. In the model of the Stroop task, $9 \%$ of the interference is located before the bottleneck and $91 \%$ is located after the bottleneck. For PWI, these percentages are different: 98\% of the retrieval-induced interference is located before the bottleneck, which is much more then the early interference in the Stroop task. This is in line with Dell'Acqua

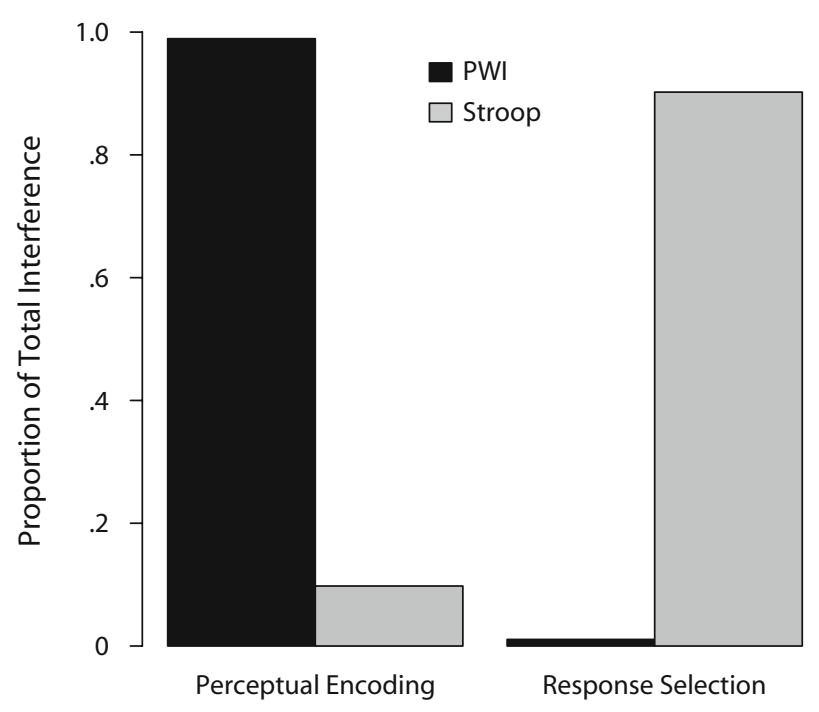

Figure 2. The proportion of interference before and after the hypothesized bottleneck. This is calculated as the proportion in each stage of the memory retrieval time for the incongruent trials minus the memory retrieval time for the control trials. 
et al.'s (2007) conclusions, because more interference in the PWI simulation is indeed located early in the processing stream.

Simulation 1 shows how a single cognitive model can account for PWI and the Stroop effect. Although this result has been obtained before (e.g., Cohen et al., 1990; Roelofs, 2003), the model presented here provides insights in the temporal distribution of the interference patterns. The model assumes that interference is a consequence of competition during memory retrieval, and shows that different processing speeds for different stimulus types (line drawings vs. easily identifiable colors) mediate the competition in subsequent stages of the task.

The ability to model both a Stroop task and a PWI task with a single model supports the view that both tasks are manifestations of the same interference process (e.g., MacLeod, 1991). By studying the memory retrieval times in different processing stages, we showed that different stimulus features may lead to a different temporal distribution of the interference patterns. This result leaves the puzzling observation by Dell'Acqua et al. (2007) that Stroop and PWI behave differently under PRP conditions. In the next section, we will extend our model to account for the PRP experiments by Fagot and Pashler (1992) and Dell'Acqua et al. (2007). We will demonstrate that a difference in stimulus features between colors and pictures may lead to different behavior in the Stroop and PWI tasks under PRP conditions.

\section{Simulation 2: A Cognitive Model of Interference During PRP}

The cognitive model presented above can account for prototypical Stroop and PWI data sets (M. O. Glaser \& Glaser, 1982, Experiment 1, SOA $=0$ for the Stroop ef- fect; W. R. Glaser \& Düngelhoff, 1984, Experiment 1, $\mathrm{SOA}=0$ for PWI). Moreover, analysis of the locus of interference in the model suggested that the magnitude of the interference effects differed between stages of the model as well as between tasks. To demonstrate that the different loci of interference may appear as different response latencies under PRP conditions, we added PRP conditions to the model. With this extension, we can assess whether the results of Fagot and Pashler (1992) and Dell'Acqua et al. (2007) can indeed be explained by assuming that PWI and Stroop interference share a common mechanism but differ in the loci in which they are manifested.

\section{Model of Fagot and Pashler (1992, Experiment 7)}

Following the experimental setup by Fagot and Pashler (1992), each trial started with a tone classification task. In this task, the participants were instructed to classify presented tones as either having a low or a high pitch by pressing one of two buttons. This additional task was added to the model using ACT-R's standard auditory perception module. As soon as the auditory system perceived a tone, a retrieval was initiated for a tone-to-button mapping. Finally, the model made a motor response to press the correct button. The model's processing of the Stroop stimulus only commenced after the response to the tone was selected, as was required according to the instructions provided to the participants. (Note that this does not mean that the buttonpress had to be finished, but that the information to be sent to the motor system had become available.) As in the Fagot and Pashler experiment, the delays between tone onset and Stroop stimulus were $-50,50$, 150 , and $450 \mathrm{msec}$. All other aspects of the model were kept constant, apart from the estimation of a fixed inter-
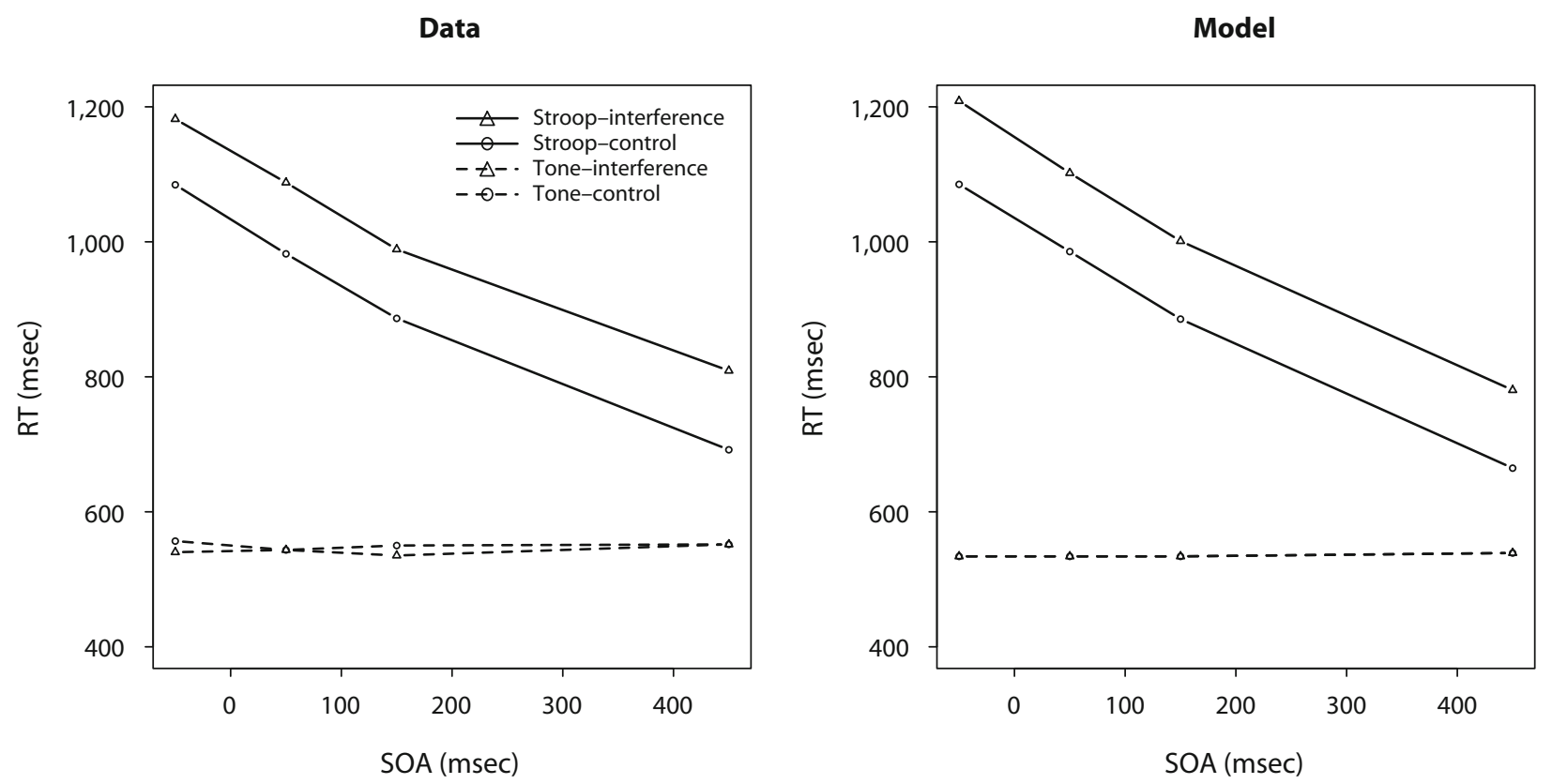

Figure 3. Model fit to the data of Fagot and Pashler (1992). Stroop denotes responses on the Stroop task, Tone denotes responses on the tone classification task. 

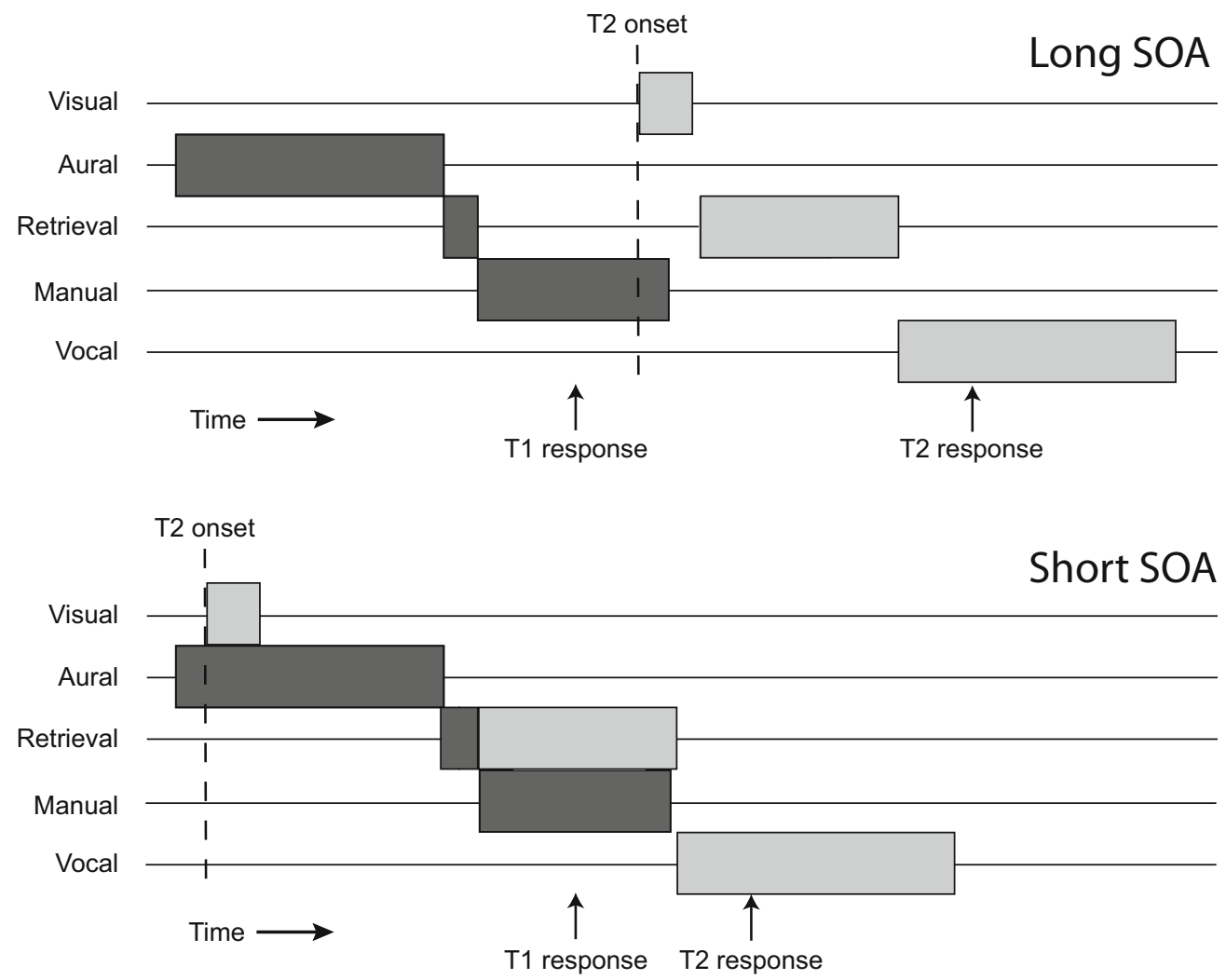

Figure 4. Gant diagrams of the model's processing of the tone classification task (dark gray) and the Stroop task (light gray). T2 onset indicates the onset of the Stroop stimulus. Each line denotes activity in a specialized module, indicated on the left. In the short SOA condition, retrieval of the appropriate response in the Stroop task has to wait until the retrieval module is available. The arrows indicate the responses for the two tasks.

cept for voice-key responses. To account for differences in naming speed between this experiment and the previously modeled data sets, we estimated the voice-key response parameter at $167 \mathrm{msec}$ (cf. Meyer \& Kieras, 1997a; Salvucci \& Taatgen, 2008).

Simulation results and discussion. The model fits of the PRP Stroop experiment (Fagot \& Pashler, 1992, Experiment 7) are presented in Figure 3. The model shows the same latency effects as Fagot and Pashler observed $\left(\mathrm{RMSE}=16 \mathrm{msec}, R^{2}=1.00\right)$. First, latency in the tone classification task is not affected by the interval between tone and the combined picture-word stimulus. In discussion of their article, Fagot and Pashler attribute this to the instruction given to the participants to always respond to the tone first and to the Stroop stimulus second. We simulated this in the model by requiring a response to be selected for the tone before the model could commence with the response selection for the Stroop task.

Second, the model shows a decrease in RT as a function of an increasing SOA, similar to the typical PRP effect found in the data. The model achieves this because as the interval between the two tasks increases, the probability that both tasks need one of the ACT-R modules at the same time decreases. As only one task can use a module at a certain time, one of the tasks has to wait when two parallel re- quests are made to a module. Also, as the interval between the tasks increases, the delay due to the task instruction regarding the response order is less; that is, if the Stroop stimulus is presented later (i.e., when the SOA is longer), the delay for the response is shorter. This is illustrated in Figure 4, which presents Gant diagrams of the modulespecific processing for both tasks. The dark gray bars represent the tone classification task, and the light gray bars represent the Stroop task. In the upper panel of Figure 4, the SOA between the tasks is long; this is reflected by the late activity in the visual module of the model. In the lower panel, the SOA is short. Initially, the Stroop stimulus is perceived, as indicated by the activity in the visual module. However, the model withholds further Stroop processing until the response in the tone classification task has been initiated. Thus, parallel to activity in the manual module, representing the buttonpress in response to the tone classification, the model initiates response selection in the Stroop task. After the model responses, both the manual and the vocal module remain occupied for a small period of time. This reflects finalizing the response-related motor actions. For instance, after a buttonpress, the model has to retract the finger and move it to the default position.

Finally, the model shows no effect of SOA on the size of the Stroop effect, which has been interpreted as evidence 


\section{Data}

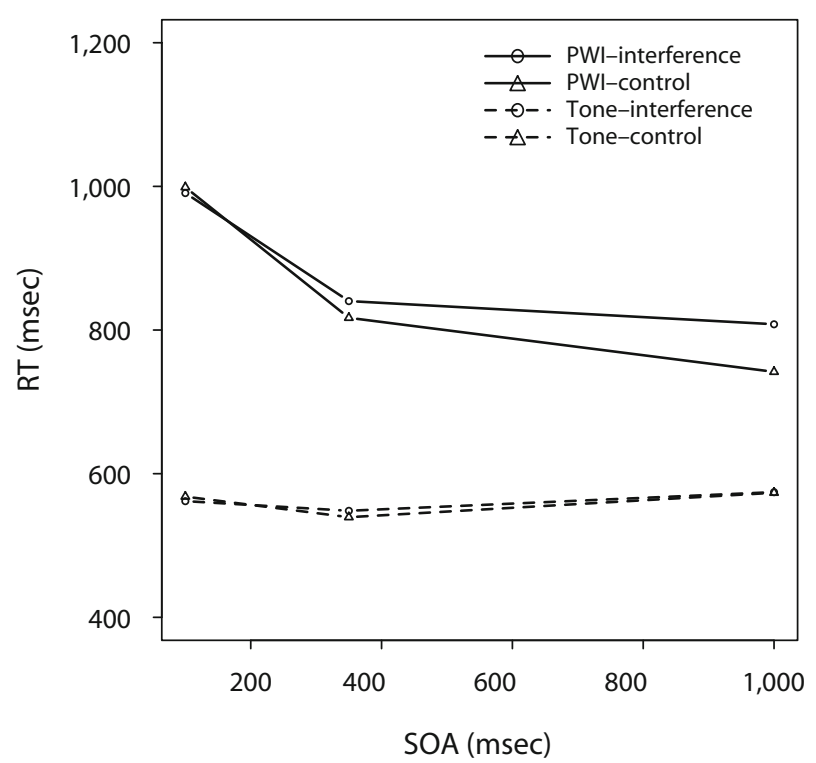

Model

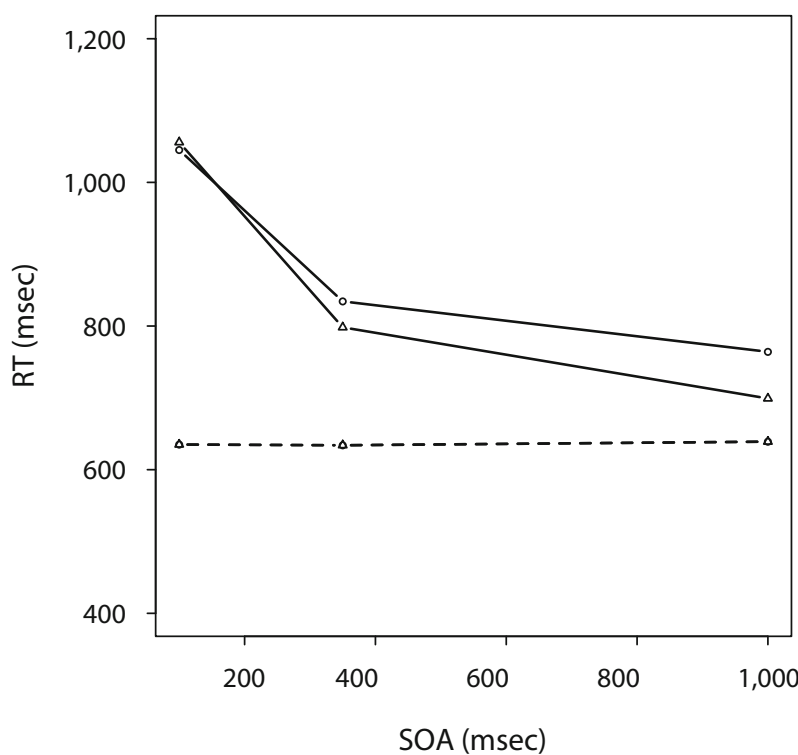

Figure 5. Model fit to the data of Dell'Acqua et al. (2007). PWI denotes responses on the PWI task, Tone denotes responses on the tone classification task.
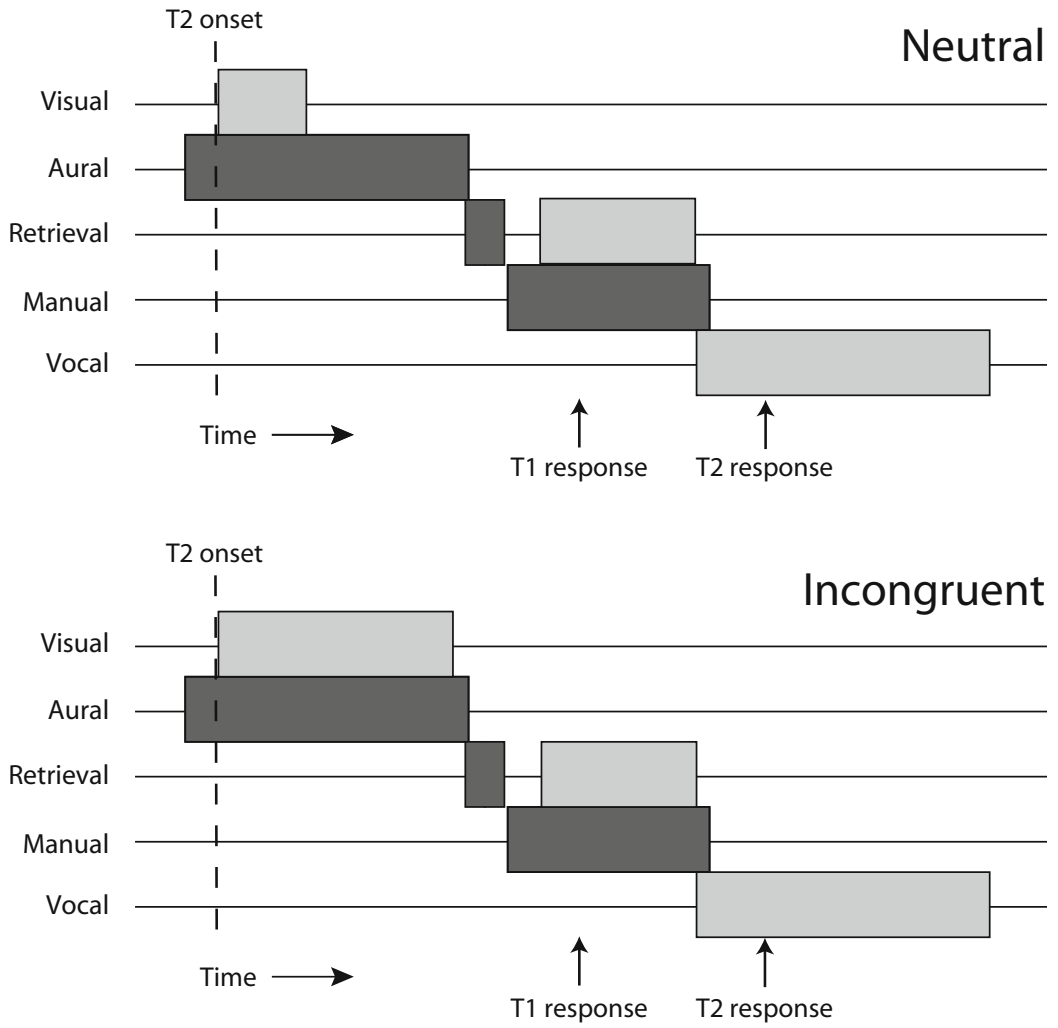

Figure 6. Gant diagrams of the model's processing of the tone classification task (dark gray) and the PWI task (light gray). Each line denotes activity in a specialized module, indicated on the left. The processing times of the PWI task are the same, regardless of the duration of the perceptual encoding stage, manifested as activity in the visual module. The arrows indicate the responses for the two tasks. 
for a late locus of the Stroop effect (Dell'Acqua et al., 2007; Fagot \& Pashler, 1992). This is because the interference in the Stroop task is mainly located in the response selection stage (see Figure 2), which does not overlap with the tone classification task.

\section{Model of Dell'Acqua et al. (2007)}

The model of the Dell'Acqua et al. (2007) experiment is a direct combination of the PWI model described under Simulation 1 and the PRP aspects described above. To match Dell'Acqua et al.'s (2007) experiment, we changed the SOAs to 100,350 , and $1,000 \mathrm{msec}$ and extended the tone classification task to three options to mirror their exact experimental setup. The single free parameter, the voice-key intercept, was estimated at $212 \mathrm{msec}$.

Simulation results and discussion. The model again found no effect of SOA on tone classification latency and the PRP effect (manifested as decreased RTs on the PWI stimulus as SOA increases). In addition, this time the model also shows an increase in interference with increased SOA (Figure 5, RMSE $=61 \mathrm{msec}, R^{2}=1.00$ ), which is in line with the result from Dell'Acqua et al. (2007). Because the interference is primarily expressed during the perceptual encoding stage, postponement of the response on the PWI stimulus (which happens for short SOAs) results in a decrease (or disappearance) of the interference effect. This is depicted in the Gant diagram in Figure 6. The upper panel shows the presentation of a neutral word-picture pair, resulting in fast perceptual encoding. However, although all information is available for further processing, production rules associated with the PWI task will not be selected because of the instruction to first finish the tone classification task. In the lower panel of Figure 6, an incongruent wordpicture pair is presented, which results in a longer perceptual encoding stage. However, as the PWI production rules have to wait for the tone task, this increased processing time does not affect the response latency. Of course, if the SOA is long, the interference effect is still apparent in the PWI response latency, because the increased latency in the perceptual system is not absorbed in the delayed response.

\section{GENERAL DISCUSSION}

In this study, we reconciled the empirical findings of Dell'Acqua et al. (2007) with the vast body of evidence toward a one-mechanism theory (as reviewed by MacLeod, 1991). Using computational cognitive modeling, we demonstrated that it is possible to account for both Stroop and PWI effects in a single model in which both interference effects are caused by the same process, even though they appear to be located in different stages. Besides this point, the model contributes to other discussions as well.

The first discussion our modeling results contribute to relates to theoretical accounts of the PRP effect. The model implemented the PRP effects by assuming an adaptive executive control structure (Meyer \& Kieras, 1997b) that controls the order in which the two responses are made. After the perceptual encoding stage of the second task (in this case, encoding of the Stroop or the PWI stimulus), the control structure locks out execution of the second task until the response of the first task has been initiated. This assumption reflects the typical task instructions given in PRP tasks, in which either participants are instructed to respond to the first task before the second task, or the importance of the first task is stressed. In the Stroop PRP and PWI PRP experiments conducted by Fagot and Pashler (1992) and Dell'Acqua et al. (2007), the correct response order was stressed as well.

Although the model diverges in this respect from the standard central bottleneck model of three stages (input, bottleneck, output; e.g, Ferreira \& Pashler, 2002; McCann \& Johnston, 1992; Pashler, 1994; Welford, 1967, 1980), it does not refute the logic of the experimental PRP design. The executive control structure creates a cognitive slack time as well, in which the initial interference can be absorbed, similar to the central bottleneck theory. However, the models presented here do support the view that such a bottleneck is not a necessary assumption for the PRP effect (Meyer \& Kieras, 1997b).

Because the model implements the executive control theory of the PRP effect, we are in a unique position to predict behavior in PRP tasks in which response order is not stressed. If the model's executive control structure is relaxed - that is, if the model is not constrained in the response order - the model's behavior does not show a PRP effect. Indeed, dual-task experiments have been conducted in which response order was not a factor, and these do not display the PRP effect (e.g., Greenwald \& Shulman, 1973; Hazeltine, Teague, \& Ivry, 2002; Schumacher et al., 1999; Schumacher et al., 2001; but see Levy \& Pashler, 2001; Ruthruff, Pashler, \& Klaassen, 2001, for opposing evidence). One caveat, however, is that the participants in these studies were trained to perform the task concurrently. Therefore, practice, not response order, might have been the main cause of the decrease of the PRP effect. This explanation would be in line with experimental and model findings that show that the PRP effect also decreases with practice in a PWI variant of the task (van Maanen, van Rijn, \& Taatgen, 2009).

Another discussion related to the PWI effect is concerned with the locus of the interference. The early locus that was reported by Dell'Acqua et al. (2007) might suggest that theories that interpret PWI as a lexical selection effect (e.g., Levelt et al., 1999; Roelofs, 1992) are incorrect. This view is supported by evidence that lexical selection is subject to central processing and therefore does not take place before the central bottleneck in the mental processing stream (Ferreira \& Pashler, 2002). In contrast, our model assumes that interference is distributed over multiple stages. Depending on the task and on task instructions, lexical selection is either a key process or appears as one of the many subprocesses. This is in line with the observation that the Stroop asynchrony is specific to the verbal response modality. If manual responses are required, less emphasis is placed on the lexical selection, and Stroop asynchrony is often absent (MacLeod, 1991).

Our modeling efforts also contribute to the discussion between single-stage and multiple-stage accounts 
of semantic interference. The hypothesis that semantic interference might be distributed over different processing stages is not new (e.g., McClelland, 1979). Multiple studies (e.g., De Houwer, 2003; Janssen, Schirm, Mahon, \& Caramazza, 2008; Risko et al., 2006; Schmidt \& Cheesman, 2005; Van Veen \& Carter, 2005) show that the locus of interference is not fixed for a particular phenomenon. Following the assumptions underlying our model, we postulate that semantic interference is associated with a particular subprocess: the retrieval of declarative knowledge from memory. This assumption is in line with studies that suggest a dissociation between stimulus-related interference and response-related interference in the Stroop task (e.g., De Houwer, 2003; Risko et al., 2006; Schmidt \& Cheesman, 2005).

\section{CONCLUSION}

The most important point that our computational modeling efforts make is that the observed difference between the Stroop and PWI effects under PRP conditions should not necessarily be interpreted as different cognitive mechanisms. Instead, it is postulated that there is no qualitative difference between the naming of colors and the naming of pictures, only a gradual difference in perception - that is, in the present setup, colors are perceived faster than pictures. This gradual difference in processing speed determines whether interference between a word and a color or picture is located to a greater extent in a perceptual stage or in a later stage. Under this assumption, a single computational model could account for the data that Dell'Acqua et al. (2007) and Fagot and Pashler (1992) observed, while doing justice to the many theoretical accounts that assume that PWI and Stroop are two manifestations of the same mechanism.

\section{AUTHOR NOTE}

The research reported here is an extension of work presented at the 30th Annual Conference of the Cognitive Science Society (van Maanen \& van Rijn, 2008). This research was financially supported by the NWO ToKeN/I2RP project (Grant 634.000.002). Correspondence concerning this article should be addressed to L. van Maanen, Department of Artificial Intelligence, University of Groningen, P.O. Box 407, $9700 \mathrm{AK}$ Groningen, The Netherlands (e-mail: leendert@ai.rug.nl).

\section{REFERENCES}

Altmann, E. M., \& Davidson, D. J. (2001). An integrative approach to Stroop: Combining a language model and a unified cognitive theory. In J. D. Moore \& K. Stenning (Eds.), Proceedings of the 23rd Annual Meeting of the Cognitive Science Society. Mahwah, NJ: Erlbaum.

ANDERSON, J. R. (2007). How can the human mind occur in the physical universe? New York: Oxford University Press.

Anderson, J. R., Bothell, D., Lebiere, C., \& Matessa, M. (1998). An integrated theory of list memory. Journal of Memory \& Language, 38, 341-380

ANDERSON, J. R., \& Milson, R. (1989). Human memory: An adaptive perspective. Psychological Review, 96, 703-719.

ANDERSON, J. R., \& SCHOOLER, L. J. (1991). Reflections of the environment in memory. Psychological Science, 2, 396-408.

Byrne, M. D., \& ANDERson, J. R. (2001). Serial modules in parallel: The psychological refractory period and perfect time-sharing. Psychological Review, 108, 847-869.

Cohen, J. D., Dunbar, K., \& McClelland, J. L. (1990). On the con- trol of automatic processes: A parallel distributed-processing account of the Stroop effect. Psychological Review, 97, 332-361.

Collins, A. M., \& LofTus, E. F. (1975). A spreading activation theory of semantic processing. Psychological Review, 82, 407-428.

De Houwer, J. (2003). On the role of stimulus-response and stimulusstimulus compatibility in the Stroop effect. Memory \& Cognition, 31, 353-359.

Dell'Acqua, R., Job, R., Peressotti, F., \& Pascali, A. (2007). The picture-word interference effect is not a Stroop effect. Psychonomic Bulletin \& Review, 14, 717-722.

Dell'Acqua, R., Lotto, L., \& Job, R. (2000). Naming times and standardized norms for the Italian PD/DPSS set of 266 pictures: Direct comparisons with American, English, French, and Spanish published databases. Behavior Research Methods, Instruments, \& Computers, 32, 588-615.

DencKla, M. B., \& Rudel, R. G. (1976). Rapid automatized naming (RAN): Dyslexia differentiated from other learning disabilities. Neuropsychologia, 14, 471-479.

Donders, F. C. (1969). On the speed of mental processes. Acta Psychologica, 30, 412-431. (Original work published 1868)

DYER, F. N. (1973). The Stroop phenomenon and its use in study of perceptual, cognitive, and response processes. Memory \& Cognition, $\mathbf{1}$, 106-120.

FaGot, C., \& Pashler, H. (1992). Making two responses to a single object: Implications for the central attentional bottleneck. Journal of Experimental Psychology: Human Perception \& Performance, 18, 1058-1079.

Ferreira, V. S., \& Pashler, H. (2002). Central bottleneck influences on the processing stages of word production. Journal of Experimental Psychology: Learning, Memory, \& Cognition, 28, 1187-1199.

Glaser, M. O., \& Glaser, W. R. (1982). Time course analysis of the Stroop phenomenon. Journal of Experimental Psychology: Human Perception \& Performance, 8, 875-894.

Glaser, W. R., \& Düngelhoff, F.-J. (1984). The time course of picture-word interference. Journal of Experimental Psychology: Human Perception \& Performance, 10, 640-654.

Glaser, W. R., \& Glaser, M. O. (1989). Context effects in Stroop-like word and picture processing. Journal of Experimental Psychology: General, 118, 13-42.

GraY, W. D. (2007a). Composition and control of integrated cognitive systems. In W. D. Gray (Ed.), Integrated models of cognitive systems (pp. 3-12). New York: Oxford University Press.

GRAY, W. D. (ED.) (2007b). Integrated models of cognitive systems. New York: Oxford University Press.

Greenwald, A. G., \& Shulman, H. G. (1973). On doing two things at once: II. Elimination of psychological refractory period. Journal of Experimental Psychology, 101, 70-76.

Hazeltine, E., Teague, D., \& Ivry, R. B. (2002). Simultaneous dualtask performance reveals parallel response selection after practice. Journal of Experimental Psychology: Human Perception \& Performance, 28, 527-545

HeNdRiKs, P., van RiJn, H., \& VAlKenier, B. (2007). Learning to reason about speakers' alternatives in sentence comprehension: A computational account. Lingua, 117, 1079-1096.

ITTI, L., \& КосH, C. (2001). Computational modeling of visual attention. Nature Reviews Neuroscience, 2, 194-203.

Janssen, N., Schirm, W., Mahon, B. Z., \& Caramazza, A. (2008). Semantic interference in a delayed naming task: Evidence for the response exclusion hypothesis. Journal of Experimental Psychology: Learning, Memory, \& Cognition, 34, 249-256.

Jolicceur, P., \& Dell'AcQua, R. (1998). The demonstration of shortterm consolidation. Cognitive Psychology, 36, 138-202.

Juvina, I., \& TAatgen, N. A. (2009). A repetition-suppression account of between-trial effects in the Stroop task. Acta Psychologica, 131, $72-84$.

KLEIN, G. S. (1964). Semantic power measured through the interference of words with color-naming. American Journal of Psychology, 77, 576-588

Kuipers, J. R., La Heis, W., \& Costa, A. (2006). A further look at semantic context effects in language production: The role of response congruency. Language \& Cognitive Processes, 21, 892-919.

Levelt, W. J. M., Roelofs, A., \& Meyer, A. S. (1999). A theory of 
lexical access in speech production. Behavioral \& Brain Sciences, 22, 1-75.

Levy, J., \& PAshler, H. (2001). Is dual-task slowing instruction dependent? Journal of Experimental Psychology: Human Perception \& Performance, 27, 862-869.

LovetT, M. C. (2005). A strategy-based interpretation of Stroop. Cognitive Science, 29, 493-524.

LucE, R. D. (1986). Response times. New York: Oxford University Press.

MACLEOD, C. M. (1991). Half a century of research on the Stroop effect: An integrative review. Psychological Bulletin, 109, 163-203.

MacLeod, C. M., \& MacDonald, P. A. (2000). Interdimensional interference in the Stroop effect: Uncovering the cognitive and neural anatomy of attention. Trends in Cognitive Sciences, 4, 383-391.

MARCEL, A. J. (1983). Conscious and unconscious perception: Experiments on visual masking and word recognition. Cognitive Psychology, 15, 197-237.

McCann, R. S., \& Johnston, J. C. (1992). Locus of the single-channel bottleneck in dual-task interference. Journal of Experimental Psychology: Human Perception \& Performance, 18, 471-484.

MCClelland, J. L. (1979). On the time relations of mental processes: Examination of systems of processes in cascade. Psychological Review, 86, 287-330.

MeYer, D. E., \& Kieras, D. E. (1997a). A computational theory of executive cognitive processes and multiple-task performance: I. Basic mechanisms. Psychological Review, 104, 3-65.

Meyer, D. E., \& Kieras, D. E. (1997b). A computational theory of executive cognitive processes and multiple-task performance: II. Accounts of psychological refractory-period phenomena. Psychological Review, 104, 749-791.

Pashler, H. (1994). Dual-task interference in simple tasks: Data and theory. Psychological Bulletin, 116, 220-244.

Risko, E. F., Schmidt, J. R., \& Besner, D. (2006). Filling a gap in the semantic gradient: Color associates and response set effects in the Stroop task. Psychonomic Bulletin \& Review, 13, 310-315.

Roelofs, A. (1992). A spreading-activation theory of lemma retrieval in speaking. Cognition, 42, 107-142.

RoELOFS, A. (2003). Goal-referenced selection of verbal action: Modeling attentional control in the Stroop task. Psychological Review, 110, $88-125$.

Rossion, B., \& Pourtols, G. (2004). Revisiting Snodgrass and Vanderwart's object pictorial set: The role of surface detail in basic-level object recognition. Perception, 33, 217-236.

Ruthruff, E., Pashler, H. E., \& Klaassen, A. (2001). Processing bottlenecks in dual-task performance: Structural limitation or strategic postponement? Psychonomic Bulletin \& Review, 8, 73-80.

Salvucci, D. D., \& Taatgen, N. A. (2008). Threaded cognition: An integrated theory of concurrent multitasking. Psychological Review, 115, 101-130.

SCHMIDT, J. R., \& CHEESMAN, J. (2005). Dissociating stimulus-stimulus and response-response effects in the Stroop task. Canadian Journal of Experimental Psychology/Revue Canadienne de Psychologie Experimentale, 59, 132-138.

Schumacher, E. H., Lauber, E. J., Glass, J. M., Zurbriggen, E. I., Gmeindl, L., Kieras, D. E., \& Meyer, D. E. (1999). Concurrent response-selection processes in dual-task performance: Evidence for adaptive executive control of task scheduling. Journal of Experimental Psychology: Human Perception \& Performance, 25, 791-814.

Schumacher, E. H., Seymour, T. L., Glass, J. M., Fencsik, D. E., Lauber, E. J., Kieras, D. E., \& Meyer, D. E. (2001). Virtually perfect time sharing in dual-task performance: Uncorking the central cognitive bottleneck. Psychological Science, 12, 101-108.

STERnBerG, S. (1969). The discovery of processing stages: Extensions of Donders' method. Acta Psychologica, 30, 276-315.

Stroop, J. R. (1935). Studies of interference in serial verbal reactions. Journal of Experimental Psychology, 18, 643-662.
TaAtgen, N. A., \& Anderson, J. R. (2002). Why do children learn to say "Broke"? A model of learning the past tense without feedback. Cognition, 86, 123-155.

TaAtgen, N. A., \& Anderson, J. R. (2008). Constraints in cognitive architectures. In R. Sun (Ed.), Handbook of computational psychology (pp. 170-185) New York: Cambridge University Press.

Taatgen, N. A., Juvina, I., Schipper, M., Borst, J. P., \& Martens, S. (2009). Too much control can hurt: A threaded cognition model of the attentional blink. Cognitive Psychology, 59, 1-29.

TAatgen, N. A., van RiJn, H., \& Anderson, J. R. (2007). An integrated theory of prospective time interval estimation: The role of cognition, attention, and learning. Psychological Review, 114, 577-598.

Telford, C. W. (1931). The refractory phase of voluntary and associative responses. Journal of Experimental Psychology, 14, 1-36.

Treisman, A. M., \& Gelade, G. (1980). A feature-integration theory of attention. Cognitive Psychology, 12, 97-136.

van MaAnen, L. (2009). Context effects on memory retrieval: Theory and applications. Unpublished doctoral dissertation, University of Groningen, Groningen.

van MaAnen, L., \& van Risn, H. (2007a). Accounting for subliminal priming in ACT-R. In R. L. Lewis, T. A. Polk, \& J. E. Laird (Eds.), Proceedings of the 8th International Conference on Cognitive Modeling (pp. 1-6). New York: Psychology Press.

van MAANEN, L., \& VAN RiJn, H. (2007b). An accumulator model of semantic interference. Cognitive Systems Research, 8, 174-181.

VAN MAANEN, L., \& VAN RIJN, H. (2008). The picture-word interference effect is a Stroop effect after all. In V. Sloutsky, B. Love, \& K. McRae (Eds.), Proceedings of the 30th Annual Meeting of the Cognitive Science Society (pp. 645-650). Austin, TX: Cognitive Science Society.

van Maanen, L., van Risn, H., \& Taatgen, N. A. (2009). Accumulators in context: An integrated theory of context effects on memory retrieval. Manuscript submitted for publication.

van RIJ, J., van RIJn, H., \& HENdRIKs, P. (2009). Cognitive architectures and language acquisition: A case study in pronoun comprehension. Manuscript submitted for publication.

van RiJn, H., \& Anderson, J. R. (2003). Modeling lexical decision as ordinary retrieval. In F. Detje, D. Dörner, \& H. Schaub (Eds.), Proceedings of the Fifth International Conference on Cognitive Modeling (pp. 207-212). Bamberg: Universitätsverlag Bamberg.

van RiJn, H., \& ANDERSON, J. R. (2004). The pseudohomophone baseword frequency effect explained. Paper presented at the Architectures and Mechanisms for Language Processing Conference (AMLaP), Aix-en-Provence.

van RiJn, H., \& TaAtgen, N. A. (2008). Timing of multiple overlapping intervals: How many clocks do we have? Acta Psychologica, 129, 365-375.

VAn Selst, M., \& Joliceur, P. (1997). Decision and response in dualtask interference. Cognitive Psychology, 33, 266-307.

VAN Veen, V., \& CARTER, C. S. (2005). Separating semantic conflict and response conflict in the Stroop task: A functional MRI study. NeuroImage, 27, 497-504

Vukovic, R. K., Wilson, A. M., \& Nash, K. K. (2004). Naming speed deficits in adults with reading disabilities: A test of the double-deficit hypothesis. Journal of Learning Disabilities, 37, 440-450.

Welford, A. T. (1967). Single-channel operation in brain. Acta Psychologica, 27, 5-22.

WeLFord, A. T. (1980). The single-channel hypothesis. In A. T. Welford (Ed.), Reaction times (pp. 215-252). London: Academic Press.

Wolfe, J. M. (1994). Guided Search 2.0: A revised model of visual search. Psychonomic Bulletin \& Review, 1, 202-238.

(Manuscript received November 6, 2008; revision accepted for publication July 15,2009 .) 